\title{
Marker-trait associations for agronomic traits in soybean harvested in Kazakhstan
}

\author{
Zatybekov A. ${ }^{1}$, Doszhanova B. ${ }^{1}$, Abugalieva S. ${ }^{1}$, Didorenko S. ${ }^{2}$, \\ Gerasimova Y. ${ }^{3}$, Sidorik I. ${ }^{4}$, Turuspekov Y. ${ }^{1 *}$ \\ ${ }^{1}$ Institute of Plant Biology and Biotechnology, Almaty, Kazakhstan \\ ${ }^{2}$ Kazakh Research Institute of Agriculture, Almaty region, Kazakhstan \\ ${ }^{3}$ East Kazakhstan Research Institute of Agriculture, Ust-Kamenogorsk region, Kazakhstan \\ ${ }^{4}$ Kostanaiskyi Research Institute of Agriculture, Kostanai region, Kazakhstan \\ *e-mail:yerlant@yahoo.com
}

Soybean is becoming one of the most important oilseed crops in Kazakhstan. In last ten years the area under soybean is expanded from 45 thousand hectares (ha) in 2006 to 120 thousand ha in 2016. The general trend of soybean expansion is from southeastern to eastern and northern regions of the country, where average temperatures are lower and growing seasons are shorter. These new soybean growing territories were poorly examined in terms of general effects on productivity level among the diverse sample of soybean accessions. Phenotypic data were collected in three separate regions of Kazakhstan and entire soybean sample was genotyped for identification of markertrait associations (MTA). In this study, the collection of 113 accessions representing five different regions of the World was planted in 2015-2016 in northern, eastern, and south-eastern regions of Kazakhstan. North American accessions showed the highest yield in four out of six trials. The collection was genotyped with 6K SNP Illumina array. 4,442 SNPs found to be polymorphic and were used for whole genome genotyping purposes. Genotyping and field data were used for GWAS (genome-wide association study). Thirty SNPs appear to be significant in 42 MTAs in six studied environments. Overall thirty SNP markers associated with time to flowering and maturation, plant height, number of fertile nodes, seeds per plant and yield were identified. Physical locations of 32 identified out of 42 total MTAs coincide well with positions of known analogous QTLs. This result indicates importance of revealed MTAs for soybean growing regions in Kazakhstan. Obtained results would serve for forming and realization of specific breeding programs towards effective adaptation and increased productivity of soybean in three different regions of Kazakhstan. 